\title{
Symbolic Formulation of Large-scale Open-loop Multibody Systems for Vibration Analysis Using Absolute Joint Coordinates*
}

\author{
Wei JIANG ${ }^{* *}$, Xuedong CHEN $^{\mathrm{a}, * * * * * *}$, Xin LUO** and Qingjiu HUANG**** \\ ** State Key Laboratory of Digital Manufacturing Equipment \& Technology \\ Huazhong University of Science and Technology, Wuhan, 430074, China \\ *** Zhengzhou University of Light Industry, Zhengzhou, 450002, China \\ E-mail: chenxd@mail.hust.edu.cn \\ **** Department of Mechanical and Control Engineering, Tokyo Institute of Technology \\ 2-12-1-I3-11 O-okayama, Meguroku, Tokyo 152-8552, Japan \\ E-mail: huang@mech.titech.ac.jp
}

\begin{abstract}
A novel symbolic formulation is presented to model dynamics of large-scale open-loop holonomic multibody systems, by using absolute joint coordinates and via matrix transformation, instead of solving constraint equations. The resulting minimal set of second-order linear ordinary differential equations (ODEs) can be used for linear vibration analysis and control directly. The ODEs are generated in three steps. Firstly, a set of linearized ODEs are formulated in terms of absolute coordinates without considering any constraint. Secondly, an overall transform matrix representing constraint topology for the entire constrained system is generated. Finally, matrices for a minimal set of ODEs for the open-loop holonomic multibody system are obtained via matrix transformation. The correctness and efficiency of the presented algorithm are verified by numerical experiments on various cases of holonomic multibody systems with different open-loop topologies, including chain topology and tree topology. It is indicated that the proposed method can significantly improve efficiency without losing computational accuracy.
\end{abstract}

Key words: Multibody Systems, Absolute Joint Coordinates, Constraints, Matrix, Vibration Analysis, Control

\section{Introduction}

Many kinds of mechanical equipments in precision engineering are very complicated in structure in order to achieve good performance in vibration isolation and/or precision positioning, e.g., optical lithography, atomic force microscopes, space telescopes, etc. Most of them can be modelled as large-scale open-loop holonomic multibody systems, which consist of large amount of rigid bodies connected by holonomic constraints with open-loop topologies, as well as arbitrary number of spatial spring-dampers. Dynamic modeling and vibration analysis based on multibody dynamics are widely recognized to be essential to design, simulation, control and optimization of these kinds of equipments ${ }^{(1)}$. Numerous efforts have focused on methodologies for formulation and solution of motion for such kinds of multibody systems ${ }^{(1 \sim 4)}$. Among them, the most widely used approach for vibration and control problems is to generate linearized equations of motion for them. The formulation is usually performed as follows: a) generate a set of differential-algebraical equations (DAEs), b) eliminate Lagrange's multipliers ${ }^{(5 \sim 8)}$, and c) linearize the resulting ODEs at a small

\footnotetext{
a Corresponding author.
} 
vicinity of the equilibrium points ${ }^{(9 \sim 12)}$. However, it is not clear which generalized coordinates should be selected to be independent in order to improve computational efficiency ${ }^{(2)}$. Furthermore, even if a proper set of independent coordinates have been selected, large amount of computational efforts needed in generation and linearization of ODEs will decrease the efficiency of formulation. It is especially severe in the cases of optimization of large-scale multibody systems that vibration analysis needs to be executed iteratively.

The coordinates used in a multibody dynamic formulation affects the structure of resulting equations of motion and the computational efficiency of solution ${ }^{(13)}$. Absolute coordinates ${ }^{(14)}$ are easy for implementation but inefficient because the number of resulting equations is much larger than that of DOFs of the system. Joint (or relative) coordinates ${ }^{(15)}$ define the relative motion of two adjacent bodies in a kinematic chain, the corresponding formulation require extra representation of the system topology which might decrease the efficiency. Absolute angular coordinates ${ }^{(15,16)}$ are reported to be simpler in form than joint coordinates for open-loop multibody systems, but they are incapable of representing relative translational motions due to prismatic joints, cylindrical joints, planar joints, etc ${ }^{(13)}$. Indirect coordinates $^{(17)}$, which measure relative motion of two bodies that are not necessarily adjacent, can be used to maximally improve the efficiency with a proper set of coordinates ${ }^{(13,18-20)}$. However, they are not straightforward and thereby are not easy to understand.

Secondly, the efficiency of formulation is also significantly affected by whether the equations of motion are generated symbolically or numerically. Symbolic formulation of multibody dynamics can speed up the solution procedure by factoring out or cancelling out some terms in the final equations in some situations ${ }^{(4,21 \sim 24)}$. Symbolic formulation can also provide better understanding of system features, particularly when the equations are formulated in matrix-vector form ${ }^{(25)}$. For vibration analysis and/or control of large-scale spatial open-loop holonomic multibody systems, symbolic formulation is a promising approach to considerably reduce complexity of formulation and improve computational efficiency of solution.

In this paper, absolute joint coordinates, an extension of absolute angular coordinates, are introduced to symbolically formulate the linearized second-order ODEs for vibration analysis of large-scale open-loop holonomic multibody systems with rotational and/or translational joints. Absolute joint coordinates represent the absolute motions of constrained body in joint-fixed reference frame using a minimal set of natural independent variables. In order to keep structural integrity and to get clear notation for derivation, all the physical parameters, i.e., mass and inertia of each body, coefficients of spatial spring-dampers, mathematical representation of joint, and spatial motion status of rigid body, are defined using matrix or vector. Upon assumption that the rotational displacements of each rigid body are small enough, e.g., systems in micro vibratory status, two fundamental linearized matrices are derived to represent transformation of micro displacements between different points on the same rigid body with respect to different reference frames. The symbolic formulation of spatial open-loop holonomic multibody systems is performed in three steps:

1) Derive linearized ODEs in terms of absolute coordinates using Lagrangian method for a multibody system without considering any constraint in the original system.

2) Generate an overall transform matrix representing constraint topology of the original open-loop holonomic multibody system based on solution of all constraint equations.

3) Perform matrix transformation to formulate a minimal set of second-order ODEs using absolute joint coordinates for the original open-loop holonomic multibody system.

Numerical experiments indicate that the proposed absolute joint coordinates are applicable to model and analyse open-loop holonomic multibody systems containing both rotational and translational joints. More important, the proposed method can greatly improve efficiency without losing computational accuracy comparing with commercial software ADAMS, especially for large-scale open-loop holonomic multibody systems. 


\section{Free Multibody System Formulation}

\subsection{Definition for spatial multibody systems}

A spatial open-loop holonomic multibody system typically consists of $n$ free rigid bodies, probably connected by at most one joint and/or arbitrary number of spatial spring-dampers between two different rigid bodies. The position and orientation of a spring-damper or a joint can be arbitrarily configured. Denote the $i^{\text {th }}$ rigid body to be $\mathrm{B}_{i}$, where $i=1,2, \cdots, n$. The number of spatial spring-dampers between $\mathrm{B}_{i}$ and $\mathrm{B}_{j}$ is $s_{i j}$, where $i, j=1,2, \cdots, n$, and $i \neq j$. The $s^{t h}$ spring-damper between $\mathrm{B}_{i}$ and $\mathrm{B}_{j}$ is denoted as $\mathrm{K}_{i j s}$, where $s=0,1,2, \cdots, s_{i j}$. Worth to note that there exist at least one and at most six holonomic constraints at a joint $\mathrm{J}_{i j}$, denote the number of constraints between body $\mathrm{B}_{i}$ and $\mathrm{B}_{j}$, if any, to be $r_{i j}$, where $1 \leq r_{i j} \leq 6$.

As shown in Fig. 1, four kinds of reference frames are taken to formulate dynamics of a spatial multibody system. A global reference frame, i.e., the inertial frame, $o-x y z$ is fixed on the ground. A body-fixed reference frame, $C_{i}-x y z$, is attached to each body $\mathrm{B}_{i}$ at its center of mass (CM). For simplicity without loss of generality, the body-fixed reference frame attached to each body $\mathrm{B}_{i}$ could be defined to be parallel to the global reference frame $o-x y z$. A spring-fixed reference frame, $K_{i j s}-x^{\prime} y^{\prime} z^{\prime}$, is attached to the corresponding spring-damper $\mathrm{K}_{i j s}$. A joint-fixed reference frame, $J_{i j}-x^{\prime \prime} y^{\prime \prime} z "$, is attached to the corresponding joint $\mathrm{J}_{i j}$.

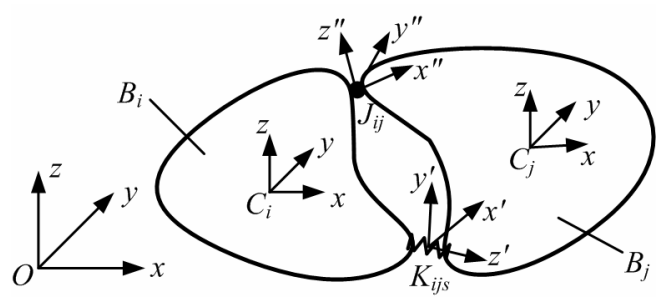

Fig. 1 Four kinds of frames used in multibody systems

The mass matrix of the $i^{\text {th }}$ rigid body $\mathrm{B}_{i}$ is given by

$$
\boldsymbol{M}_{i}=\operatorname{diag}\left(m_{i} \boldsymbol{I} \quad \boldsymbol{J}_{i}\right)
$$

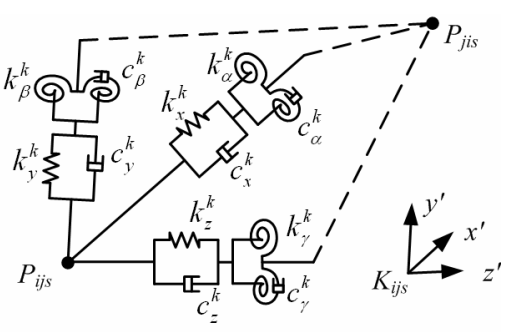

Fig. 2 Spatial spring-damper

where $m_{i}$ is the mass of $\mathrm{B}_{i}, \boldsymbol{J}_{i}$ is the inertia tensor with respect to its body-fixed reference frame $C_{i}-x y z$, and $\boldsymbol{I}$ is a $3 \times 3$ identity matrix.

The mass matrix of the multibody system under consideration can be arranged as

$$
\boldsymbol{M}=\operatorname{diag}\left(\begin{array}{llll}
\boldsymbol{M}_{1} & \boldsymbol{M}_{2} & \cdots & \boldsymbol{M}_{n}
\end{array}\right)
$$

The translational and rotational displacements at $\mathrm{CM}$ of body $\mathrm{B}_{i}$ with respect to the inertial frame $o-x y z$ can be written in vector form as

$$
\boldsymbol{x}_{i}=\left[\begin{array}{ll}
\boldsymbol{r}_{i}^{\mathrm{T}} & \boldsymbol{\theta}_{i}^{\mathrm{T}}
\end{array}\right]^{\mathrm{T}}=\left[\begin{array}{llllll}
x_{i} & y_{i} & z_{i} & \alpha_{i} & \beta_{i} & \gamma_{i}
\end{array}\right]^{\mathrm{T}}
$$

where the rotational displacements $\boldsymbol{\theta}$ are represented with Bryan angles (or Cardan angles) in rotation sequence of $x-y-z$.

The displacements relative to equilibrium points are selected as absolute coordinates for the entire system

$$
\boldsymbol{x}=\left[\begin{array}{llll}
\boldsymbol{x}_{1}^{\mathrm{T}} & \boldsymbol{x}_{2}^{\mathrm{T}} & \cdots & \boldsymbol{x}_{n}^{\mathrm{T}}
\end{array}\right]^{\mathrm{T}}
$$

Given that the rotational displacements are small enough, i.e., $\alpha, \beta, \gamma \approx 0$, the velocities and accelerations of each body can be expressed using the $1^{\text {st }}$ order and the $2^{\text {nd }}$ order differentiation of the displacements with respect to time, respectively, i.e., $\dot{\boldsymbol{x}}=\left[\begin{array}{llll}\dot{\boldsymbol{x}}_{1}^{\mathrm{T}} & \dot{\boldsymbol{x}}_{2}^{\mathrm{T}} & \cdots & \dot{\boldsymbol{x}}_{n}^{\mathrm{T}}\end{array}\right]^{\mathrm{T}}, \quad \ddot{\boldsymbol{x}}=\left[\begin{array}{llll}\ddot{\boldsymbol{x}}_{1}^{\mathrm{T}} & \ddot{\boldsymbol{x}}_{2}^{\mathrm{T}} & \cdots & \ddot{\boldsymbol{x}}_{n}^{\mathrm{T}}\end{array}\right]^{\mathrm{T}}$.

As shown in Fig.2, the spatial spring-damper means that both springs and dampers work in all three translational and three rotational directions. The stiffness and damping matrices of $\mathrm{K}_{i j s}$ are defined in spring-fixed reference frame $K_{i j s}-x y z$ as

$$
\begin{aligned}
\boldsymbol{K}_{i j s}^{k} & =\operatorname{diag}\left(\begin{array}{llllll}
k_{x i j s}^{k} & k_{y j j s}^{k} & k_{z i j s}^{k} & k_{\alpha i j s}^{k} & k_{\beta i j s}^{k} & k_{\gamma i j s}^{k}
\end{array}\right) \\
\boldsymbol{C}_{i j s}^{k} & =\operatorname{diag}\left(\begin{array}{llllll}
c_{x i j s}^{k} & c_{y j j s}^{k} & c_{z i j s}^{k} & c_{\alpha i j s}^{k} & c_{\beta i j s}^{k} & c_{\gamma i j s}^{k}
\end{array}\right)
\end{aligned}
$$

The acting points of $\mathrm{K}_{i j s}$ upon $\mathrm{B}_{i}$ and $\mathrm{B}_{j}$ are $\mathrm{P}_{i j s}$ and $\mathrm{P}_{j i s}$, respectively. The position of $\mathrm{P}_{i j s}$ with respect to $C_{i}-x y z$ is marked as $\underline{\boldsymbol{x}}_{i j s}^{c}=\left[\begin{array}{lll}x_{j i s}^{c} & \underline{y}_{i j s}^{c} & \underline{\underline{c}}_{j j}^{c}\end{array}\right]^{\mathrm{T}}$, and the position of $\mathrm{P}_{j i s}$ with respect 
to $C_{i}-x y z$ is marked as $\underline{\boldsymbol{x}}_{j i s}^{c}=\left[\begin{array}{lll}x_{j i s}^{c} & y_{j i s}^{c} & \underline{z}_{j i s}^{c}\end{array}\right]^{\mathrm{T}}$. The posture of $\mathrm{K}_{i j s}$ is defined by $\boldsymbol{A}_{i j s}^{c k}$, which is the direction cosine matrix relating the body-fixed reference frame $C_{i}-x y z$ to the spring-fixed reference frame $K_{i j s}-x y z$.

The force acting upon $\mathrm{B}_{j}$ that is generated by spatial spring-damper $\mathrm{K}_{i j s}$ can be formulated in spring-fixed reference frame as

$$
\boldsymbol{f}_{k i j s}^{k}=\boldsymbol{K}_{i j s}^{k}\left(\boldsymbol{x}_{\mathrm{P} i j s}^{k}-\boldsymbol{x}_{\mathrm{P} j i s}^{k}\right)+\boldsymbol{C}_{i j s}^{k}\left(\dot{\boldsymbol{x}}_{\mathrm{P} i j s}^{k}-\dot{\boldsymbol{x}}_{\mathrm{P} j i s}^{k}\right)
$$

Where $\boldsymbol{x}_{\mathrm{P} i j s}^{k}$ and $\dot{\boldsymbol{x}}_{\mathrm{P} i j s}^{k}$ are the displacement and velocity vector of point $\mathrm{P}_{i j s}$ with respect to $K_{i j s}-x y z$, respectively. $\quad \boldsymbol{x}_{\mathrm{P} i j s}^{k}$ and $\dot{\boldsymbol{x}}_{\mathrm{P} j i s}^{k}$ are the displacement and velocity vector of point $\mathrm{P}_{j i s}$ with respect to $K_{i j s}-x y z$, respectively.

\subsection{Linearization of displacements transformation}

In order to calculate the potential energy in all springs, the differences between displacements of a pair of acting points for each spring must be figured out. This leads to transformation of displacements of each body at spring acting point from the global reference frame to the spring-fixed reference frame. The calculation can be decomposed into two steps: firstly formulate the displacements at spring acting point with respect to the global reference frame using displacements at its $\mathrm{CM}$, and then transform the displacements from the global reference frame to the spring-fixed reference frame.

The relationship between reference frames before and after rotation can be represented by the direction cosine matrix $\boldsymbol{A}$, which is determined by rotational angle $\boldsymbol{\theta}=\left[\begin{array}{lll}\alpha & \beta & \gamma\end{array}\right]^{\mathrm{T}}$ as

$$
\boldsymbol{A}=\left[\begin{array}{ccc}
\mathrm{C}_{\beta} \mathrm{C}_{\gamma} & -\mathrm{C}_{\beta} \mathrm{S}_{\gamma} & \mathrm{S}_{\beta} \\
\mathrm{S}_{\alpha} \mathrm{S}_{\beta} \mathrm{C}_{\gamma}+\mathrm{C}_{\alpha} \mathrm{S}_{\gamma} & -\mathrm{S}_{\alpha} \mathrm{S}_{\beta} \mathrm{S}_{\gamma}+\mathrm{C}_{\alpha} \mathrm{C}_{\gamma} & -\mathrm{S}_{\alpha} \mathrm{C}_{\beta} \\
-\mathrm{C}_{\alpha} \mathrm{S}_{\beta} \mathrm{C}_{\gamma}+\mathrm{S}_{\alpha} \mathrm{S}_{\gamma} & \mathrm{C}_{\alpha} \mathrm{S}_{\beta} \mathrm{S}_{\gamma}+\mathrm{S}_{\alpha} \mathrm{C}_{\gamma} & \mathrm{C}_{\alpha} \mathrm{C}_{\beta}
\end{array}\right]
$$

Where denote $\mathrm{S}_{\mu}=\sin \mu$ and $\mathrm{C}_{\mu}=\cos \mu(\mu=\alpha, \beta, \gamma)$. Given the condition that rotational displacements are small enough, i.e., $\alpha, \beta, \gamma \approx 0$, which is satisfied well for vibration analysis, Eq.(2) can be linearized as

$$
\boldsymbol{A} \approx\left[\begin{array}{ccc}
1 & -\gamma & \beta \\
\gamma & 1 & -\alpha \\
-\beta & \alpha & 1
\end{array}\right]
$$

Let $\mathrm{P}$ be a fixed point on the body, and the relative positions of $\mathrm{P}$ with respect to the $\mathrm{CM}$ of the body is marked as $\underline{\boldsymbol{x}}_{\mathrm{CP}}^{c}=\left[\begin{array}{lll}x_{\mathrm{CP}}^{c} & \underline{y}_{\mathrm{CP}}^{c} & \underline{z}_{\mathrm{CP}}^{c}\end{array}\right]^{\mathrm{T}}$. According to the concept of rigid body, the rotational displacements of different points on the same rigid body are equal to each other. It means that the translational and rotational displacements of the body can be expressed at point $\mathrm{P}$ with respect to $o-x y z$ as

$$
\boldsymbol{x}_{\mathrm{P}}=\left[\begin{array}{c}
\boldsymbol{r}_{\mathrm{P}} \\
\boldsymbol{\theta}_{\mathrm{P}}
\end{array}\right]=\left[\begin{array}{c}
\boldsymbol{r}_{\mathrm{C}}+(\boldsymbol{A}-\boldsymbol{I}) \underline{\boldsymbol{x}}_{\mathrm{CP}}^{c} \\
\boldsymbol{\theta}_{\mathrm{C}}
\end{array}\right]
$$

Substitute Eq.(3) into Eq.(4) and yield

$$
\boldsymbol{x}_{\mathrm{P}}=\boldsymbol{T}_{\mathrm{CP}} \boldsymbol{x}_{\mathrm{C}}=\left[\begin{array}{cc}
\boldsymbol{I} & \boldsymbol{U}_{\mathrm{CP}} \\
\boldsymbol{0} & \boldsymbol{I}
\end{array}\right] \boldsymbol{x}_{\mathrm{C}}
$$

where $\boldsymbol{x}_{\mathrm{C}}$ the displacements vector at $\mathrm{CM}$ as specified in Eq.(1), and $\boldsymbol{U}_{\mathrm{CP}}$ a skew symmetric matrix determined by $\underline{\boldsymbol{x}}_{\mathrm{CP}}^{c}$ as

$$
\boldsymbol{U}_{\mathrm{CP}}=\left[\begin{array}{ccc}
0 & \underline{z}_{\mathrm{CP}}^{c} & -\underline{y}_{\mathrm{CP}}^{c} \\
-\underline{z}_{\mathrm{CP}}^{c} & 0 & \underline{x}_{\mathrm{CP}}^{c} \\
\underline{y}_{\mathrm{CP}}^{c} & -\underline{x}_{\mathrm{CP}}^{c} & 0
\end{array}\right]
$$

It indicates that the relationship of displacements between different points on a same rigid body can be formulated using a constant matrix which is definitely determined by relative positions between these two points.

Assume that there is another reference frame $v-x y z$. Let $\boldsymbol{A}^{o v}$ be the direction cosine matrix representing the rotation from $o-x y z$ to $v-x y z$. The translational and rotational displacements can both be regarded as vectors in a space, and their transformations between different reference frames can be expressed using $\boldsymbol{A}^{o v}$ as follows: 


$$
\boldsymbol{x}_{\mathrm{C}}^{v}=\left[\begin{array}{l}
\boldsymbol{r}_{\mathrm{C}}^{v} \\
\boldsymbol{\theta}_{\mathrm{C}}^{v}
\end{array}\right]=\left[\begin{array}{cc}
\boldsymbol{A}^{o v \mathrm{~T}} & \boldsymbol{0} \\
\boldsymbol{0} & \boldsymbol{A}^{o v \mathrm{~T}}
\end{array}\right]\left[\begin{array}{l}
\boldsymbol{r}_{\mathrm{C}}^{o} \\
\boldsymbol{\theta}_{\mathrm{C}}^{o}
\end{array}\right]=\boldsymbol{R}^{o v} \boldsymbol{x}_{\mathrm{C}}^{o}
$$

It means that the transformation of displacements at one identical point from with respect to one reference frame to another can be expressed using the transformation matrix $\boldsymbol{R}$ specified in Eq.(7).

Accordingly, the displacements at spring acting point $\mathrm{P}_{i j s}$ with respect to $K_{i j s}-x y z$ can be expressed by displacements at $\mathrm{CM}$ of the same body with respect to $o-x y z$

$$
\boldsymbol{x}_{i j s}^{k}=\boldsymbol{R}_{i j s}^{c k} \boldsymbol{T}_{i j s} \boldsymbol{x}_{i}
$$

where $\boldsymbol{T}_{i j s}$ can be formulated using $\underline{x}_{i j s}^{c}$ according to Eq.(5)-(6), and $\boldsymbol{R}_{i j s}^{c k}$ can be formulated using $\boldsymbol{A}_{i j s}^{\text {ck }}$ according to Eq.(7).

\subsection{Formulation of free multibody systems}

The total kinetic energy of the system, i.e., the sum of kinetic energy of all rigid bodies of the system, is calculated as

And we obtain that

$$
T=\frac{1}{2} \dot{\boldsymbol{x}}^{\mathrm{T}} \boldsymbol{M} \dot{\boldsymbol{x}}=\sum_{i=1}^{n} \frac{1}{2} \dot{\boldsymbol{x}}_{i}^{\mathrm{T}} \boldsymbol{M}_{i} \dot{\boldsymbol{x}}_{i}
$$

$$
\frac{\mathrm{d}}{\mathrm{d} t} \frac{\partial T}{\partial \dot{\boldsymbol{x}}_{i}^{\mathrm{T}}}=\frac{1}{2}\left(\boldsymbol{M}_{i}+\boldsymbol{M}_{i}^{\mathrm{T}}\right) \ddot{\boldsymbol{x}}_{i}=\boldsymbol{M}_{i} \ddot{\boldsymbol{x}}_{i}
$$

The small deformation of spring $\mathrm{K}_{i j s}$ can be regarded as difference of displacements between $\mathrm{P}_{i j s}$ and $\mathrm{P}_{j i s}$ with respect to $K_{i j s}-x y z$, i.e.,

$$
\Delta \boldsymbol{x}_{i j s}^{k}=\boldsymbol{R}_{i j / \boldsymbol{S}_{i j s}}^{c k} \boldsymbol{x}_{i}-\boldsymbol{R}_{i j s}^{c k} \boldsymbol{T}_{j i s} \boldsymbol{x}_{j}
$$

Set the potential energy of the system at equilibrium positions to be zero. Then the potential energy of the entire system with respect to equilibrium points is expressed as the sum of changes in geopotential and those in all springs

$$
U=\sum_{i=1}^{n} \boldsymbol{g}_{i}^{\mathrm{T}} \boldsymbol{M}_{i} \boldsymbol{x}_{i}+\sum_{i=0}^{n-1} \sum_{j=i+1}^{n} \sum_{s=1}^{s_{i j}} \frac{1}{2}\left(\Delta \boldsymbol{x}_{i j s}^{k}\right)^{\mathrm{T}} \boldsymbol{K}_{i j s}^{k} \Delta \boldsymbol{x}_{i j s}^{k}
$$

where $\boldsymbol{g}_{i}=\left[\begin{array}{llllll}0 & -g & 0 & 0 & 0 & 0\end{array}\right]^{\mathrm{T}}(i=1,2, \cdots, n)$. Substitute Eq.(8) into Eq.(9), and then partially derivate $U$ with respect to $\boldsymbol{x}_{i}^{\mathrm{T}}$, we obtain

$$
\frac{\partial U}{\partial \boldsymbol{x}_{i}^{\mathrm{T}}}=\boldsymbol{K}_{i i} \boldsymbol{x}_{i}-\sum_{j=0}^{n} \boldsymbol{K}_{i j} \boldsymbol{x}_{j}+\boldsymbol{M}_{i} \boldsymbol{g}_{i}
$$

where $\boldsymbol{K}_{i i}=\sum_{j=0}^{n} \sum_{s=1}^{s_{j}} \boldsymbol{T}_{i j s}{ }^{\mathrm{T}}\left(\boldsymbol{R}_{i j s}^{c k}\right)^{\mathrm{T}} \boldsymbol{K}_{i j s}^{k} \boldsymbol{R}_{i j s}^{c k} \boldsymbol{T}_{i j s}, \quad \boldsymbol{K}_{i j}=\sum_{s=1}^{s_{i j}} \boldsymbol{T}_{i j s}^{\mathrm{T}}\left(\boldsymbol{K}_{i j s}^{c k}\right)^{\mathrm{T}} \boldsymbol{K}_{i j s}^{k} \boldsymbol{R}_{i j s}^{c k} \boldsymbol{T}_{j i s}$.

The damping force acting on $\mathrm{B}_{i}$ can be treated as generalized force and formulated with respect to $C_{i}-x y z$ via time derivation of Eq.(8) as

$$
\boldsymbol{f}_{d i}=-\sum_{j=0}^{n} \sum_{s=1}^{s_{i j}} \boldsymbol{T}_{i j s}{ }^{\mathrm{T}}\left(\boldsymbol{R}_{i j s}^{c k}\right)^{\mathrm{T}} \boldsymbol{C}_{i j s}^{k} \Delta \dot{\boldsymbol{x}}_{i j s}^{k}=-\boldsymbol{C}_{i i} \dot{\boldsymbol{x}}_{i}+\sum_{j=0}^{n} \boldsymbol{C}_{i j} \dot{\boldsymbol{x}}_{j}
$$

where $\boldsymbol{C}_{i i}=\sum_{j=0}^{n} \sum_{s=1}^{s_{i j}} \boldsymbol{T}_{i j s}^{\mathrm{T}}\left(\boldsymbol{R}_{i j s}^{c k}\right)^{\mathrm{T}} \boldsymbol{C}_{i j s}^{k} \boldsymbol{R}_{i j s}^{c k} \boldsymbol{T}_{i j s}, \boldsymbol{C}_{i j}=\sum_{s=1}^{s_{j i}} \boldsymbol{T}_{i j s}{ }^{\mathrm{T}}\left(\boldsymbol{R}_{i j s}^{c k}\right)^{\mathrm{T}} \boldsymbol{C}_{i j s}^{k} \boldsymbol{R}_{i j s}^{c k} \boldsymbol{T}_{j i s}$.

Denote $\boldsymbol{M g}=\left[\begin{array}{llll}\left(\boldsymbol{M}_{1} \boldsymbol{g}_{1}\right)^{\mathrm{T}} & \left(\boldsymbol{M}_{2} \boldsymbol{g}_{2}\right)^{\mathrm{T}} & \cdots & \left(\boldsymbol{M}_{n} \boldsymbol{g}_{n}\right)^{\mathrm{T}}\end{array}\right]^{\mathrm{T}}$ the gravitational force vector acting on all bodies in the system, and $\boldsymbol{f}_{e}=\left[\begin{array}{llll}\boldsymbol{f}_{e 1}{ }^{\mathrm{T}} & \boldsymbol{f}_{e 2}{ }^{\mathrm{T}} \cdots & \boldsymbol{f}_{e n}{ }^{\mathrm{T}}\end{array}\right]^{\mathrm{T}}$ other external force vector acting on the system, the free multibody system follows the linearized ODEs

$$
\boldsymbol{M} \ddot{\boldsymbol{x}}+\boldsymbol{C} \dot{\boldsymbol{x}}+\boldsymbol{K} \boldsymbol{x}=\boldsymbol{f}_{e}+\boldsymbol{M g}-\boldsymbol{M g}=\boldsymbol{f}_{e}
$$

where the system damping matrix $\boldsymbol{C}$ and stiffness matrix $\boldsymbol{K}$ be of the same form as

$$
\boldsymbol{E}=\left[\begin{array}{cccc}
\boldsymbol{E}_{11} & -\boldsymbol{E}_{12} & \cdots & -\boldsymbol{E}_{1 n} \\
-\boldsymbol{E}_{21} & \boldsymbol{E}_{22} & \cdots & \vdots \\
\vdots & \vdots & \ddots & -\boldsymbol{E}_{n-1, n} \\
-\boldsymbol{E}_{n 1} & \cdots & -\boldsymbol{E}_{n, n-1} & \boldsymbol{E}_{n n}
\end{array}\right] \quad(\boldsymbol{E}=\boldsymbol{C}, \boldsymbol{K})
$$

\section{Constrained Multibody System Formulation}

\subsection{Mathematical definition of holonomic constraints}

Most of the joints that used for practical applications are modeled in terms of the so-called lower pairs, including revolute, prismatic, cylindrical, universal, spherical, and planar joints. 
Each joint reduce corresponding number of DOFs of the distal ${ }^{(13)}$ body between two connected bodies. Assume there is an ideal joint $\mathrm{J}_{a b}$ between body $\mathrm{B}_{a}$ and $\mathrm{B}_{b}$. The acting points of $\mathrm{J}_{a b}$ on $\mathrm{B}_{a}$ and $\mathrm{B}_{b}$ are marked as $\mathrm{Q}_{a b}$ and $\mathrm{Q}_{b a}$, respectively. The position of $\mathrm{Q}_{a b}$ with respect to $C_{a}-x y z$ is denoted as $\underline{\boldsymbol{x}}_{a b}^{c}=\left[\begin{array}{lll}x_{a b}^{c} & \underline{y}_{a b}^{c} & \underline{z}_{a b}^{c}\end{array}\right]^{\mathrm{T}}$. Similarly, the position of $\mathrm{Q}_{b a}$ with respect to $C_{b}-x y z$ is denoted as $\underline{\boldsymbol{x}}_{b a}^{c}=\left[\begin{array}{lll}x_{b a}^{c} & \bar{y}_{b a}^{c} & \underline{z}_{b a}^{c}\end{array}\right]^{\mathrm{T}}$. Let $\boldsymbol{A}_{a b}^{c i}$ be the direction cosine matrix relating $C_{a}-x y z$ to the joint-fixed reference frame $J_{a b}-x y z$. The displacements of $\mathrm{Q}_{a b}$ and $\mathrm{Q}_{b a}$ with respect to $J_{a b}-x y z$, i.e., absolute joint coordinates, are denoted as $\boldsymbol{x}_{a Q}^{j}$ and $\boldsymbol{x}_{b \mathrm{Q}}^{j}$. A $6 \times 6$ diagonal matrix $\boldsymbol{H}$ is introduced to formulate the constraint equations as follows

$$
\boldsymbol{H} \boldsymbol{x}_{a Q}^{j}=\boldsymbol{H} \boldsymbol{x}_{b Q}^{j}
$$

The meaning of the constraint matrix $\boldsymbol{H}$ can be explained as follows: each diagonal element of the matrix is one or zero, representing whether the DOF along the corresponding axis is constrained or not. In order to minimally reduce the number of equations for the constrained multibody system, a new matrix $\boldsymbol{D}$ is introduced for each kind of joint to extract the independent coordinates in $\boldsymbol{x}_{b Q}^{j}$, i.e., $\boldsymbol{x}_{\text {independent }}=\boldsymbol{D}^{\mathrm{T}} \boldsymbol{x}_{b \mathrm{Q}}^{j}$. Matrix $\boldsymbol{D}$ is defined in such a way that all columns, whose elements are all zeros in matrix $\boldsymbol{I}_{6}-\boldsymbol{H}$, are removed.

Constraint matrices for each kind of ideal joints are listed in Table 1.

Table 1 Mathematical library of joints

\begin{tabular}{|c|c|c|c|}
\hline Joint type & Free axes & Matrix $\boldsymbol{H}$ & Matrix $\boldsymbol{D}$ \\
\hline Fixed & none & $\boldsymbol{I}_{6}$ & null matrix \\
\hline revolute & $\gamma$ & $\operatorname{diag}\left(\begin{array}{llllll}1 & 1 & 1 & 1 & 1 & 0\end{array}\right)$ & {$\left[\begin{array}{llllll}0 & 0 & 0 & 0 & 0 & 1\end{array}\right]^{\mathrm{T}}$} \\
\hline prismatic & $z$ & $\operatorname{diag}\left(\begin{array}{llllll}1 & 1 & 0 & 1 & 1 & 1\end{array}\right)$ & {$\left[\begin{array}{llllll}0 & 0 & 1 & 0 & 0 & 0\end{array}\right]^{\mathrm{T}}$} \\
\hline cylindrical & $z, \gamma$ & $\operatorname{diag}\left(\begin{array}{llllll}1 & 1 & 0 & 1 & 1 & 0\end{array}\right)$ & {$\left[\begin{array}{llllll}0 & 0 & 1 & 0 & 0 & 0 \\
0 & 0 & 0 & 0 & 0 & 1\end{array}\right]^{\mathrm{T}}$} \\
\hline universal & $\alpha, \beta$ & $\operatorname{diag}\left(\begin{array}{llllll}1 & 1 & 1 & 0 & 0 & 1\end{array}\right)$ & {$\left[\begin{array}{llllll}0 & 0 & 0 & 1 & 0 & 0 \\
0 & 0 & 0 & 0 & 1 & 0\end{array}\right]^{\mathrm{T}}$} \\
\hline spherical & $\alpha, \beta, \gamma$ & $\operatorname{diag}\left(\begin{array}{llllll}1 & 1 & 1 & 0 & 0 & 0\end{array}\right)$ & {$\left[\begin{array}{llllll}0 & 0 & 0 & 1 & 0 & 0 \\
0 & 0 & 0 & 0 & 1 & 0 \\
0 & 0 & 0 & 0 & 0 & 1\end{array}\right]^{\mathrm{T}}$} \\
\hline planar & $x, y, \gamma$ & $\operatorname{diag}\left(\begin{array}{llllll}0 & 0 & 1 & 1 & 1 & 0\end{array}\right)$ & {$\left[\begin{array}{llllll}1 & 0 & 0 & 0 & 0 & 0 \\
0 & 1 & 0 & 0 & 0 & 0 \\
0 & 0 & 0 & 0 & 0 & 1\end{array}\right]^{\mathrm{T}}$} \\
\hline
\end{tabular}

\subsection{Formulation of open-loop multibody systems}

Generally, there might be more then one joint in a multibody system. As shown in Fig.3, the topology of constraints in multibody system can be classified into four groups: (a) scatter, (b) chain, (c) tree, and (d) closed-loop. The first three kinds can all be regarded as open-loop constrained system. It is worth to note that the spring-dampers between two nonadjacent bodies are not shown in the figure.

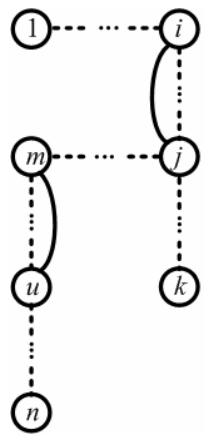

(a) Scatter

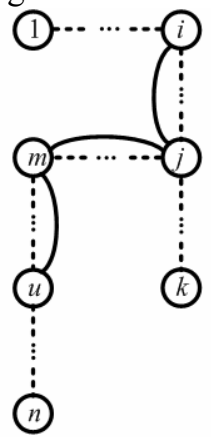

(b) Chain



(c) Tree

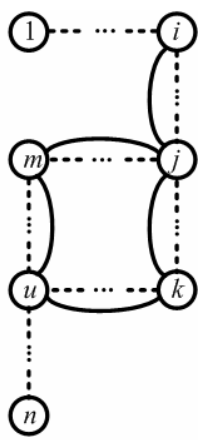

(d) Closed-loop

$$
\text { (i) }{\text { Body } \mathrm{B}_{i}} \quad \cdots \quad \text { Bodies connected by spring-dampers }
$$

Fig. 3 Topologies of constraints in multibody systems 
In this section, we explain about how to obtain a minimal set of linearized ODEs via construction and transformation of matrix for a general open-loop holonomic multibody system. For convenience of explanation, we take an example as shown in Fig. 3(c).

In Fig. 3(c), a system with $n$ rigid bodies is considered, among which $\mathrm{B}_{i}, \mathrm{~B}_{j}$ and $\mathrm{B}_{k}$ are connected with joints $\mathrm{J}_{i j}$ and $\mathrm{J}_{j k}$, serially, whereas $\mathrm{B}_{j}, \mathrm{~B}_{m}$ and $\mathrm{B}_{u}$ are connected with joints $\mathrm{J}_{j m}$ and $\mathrm{J}_{m u}$, serially. Without losing generality, assume that $1 \leq i<j<k<m<u \leq n$.

The constraint equations for joint $\mathrm{J}_{i j}$ can be written as

$$
\boldsymbol{H}_{i j} \boldsymbol{x}_{i \mathrm{Q}}^{j}=\boldsymbol{H}_{i j} \boldsymbol{R}_{i j}^{c j} \boldsymbol{T}_{i \mathrm{Q}} \boldsymbol{x}_{i}=\boldsymbol{H}_{i j} \boldsymbol{x}_{j \mathrm{Q}}^{j}
$$

where $\boldsymbol{T}_{i \mathrm{Q}}$ and $\boldsymbol{R}_{i j}^{c j}$ are generated according to Eq.(5) and Eq.(7). Using properties $\boldsymbol{T}_{\mathrm{PQ}}^{-1}=\boldsymbol{T}_{\mathrm{QP}}$ and $\boldsymbol{R}^{o v-1}=\boldsymbol{R}^{v o}$, the constraint equations can be expressed in another form

$$
\boldsymbol{x}_{j}=\boldsymbol{P}_{i j} \boldsymbol{x}_{i}+\boldsymbol{Q}_{i j} \boldsymbol{x}_{j \mathrm{Q}}^{j}
$$

where $\boldsymbol{P}_{i j}=\boldsymbol{T}_{\mathrm{Q} j} \boldsymbol{R}_{i j}^{j c} \boldsymbol{H}_{i j} \boldsymbol{R}_{i j}^{c j} \boldsymbol{T}_{i \mathrm{Q}}, \boldsymbol{Q}_{i j}=\boldsymbol{T}_{\mathrm{Q} j} \boldsymbol{R}_{i j}^{j c}\left(\boldsymbol{I}_{6}-\boldsymbol{H}_{i j}\right)$.

The constraint equations for joint $\mathrm{J}_{j k}$ can be written as

$$
\boldsymbol{x}_{k}=\boldsymbol{P}_{j k} \boldsymbol{P}_{i j} \boldsymbol{x}_{i}+\boldsymbol{P}_{j k} \boldsymbol{Q}_{i j} \boldsymbol{x}_{j \mathrm{Q}}^{j}+\boldsymbol{Q}_{j k} \boldsymbol{x}_{k \mathrm{Q}}^{j}
$$

The constraint equations for the rest joints can all be formulated recursively similar to Eq.(11). The constraint equations for the entire system can be written as

$$
\begin{gathered}
\boldsymbol{x}=\boldsymbol{B} \overline{\boldsymbol{x}} \\
\overline{\boldsymbol{x}}=\left[\begin{array}{llll}
\boldsymbol{q}_{1}{ }^{\mathrm{T}} & \boldsymbol{q}_{2}^{\mathrm{T}} & \cdots & \boldsymbol{q}_{n}{ }^{\mathrm{T}}
\end{array}\right]^{\mathrm{T}}
\end{gathered}
$$

where $\boldsymbol{q}_{\varepsilon}^{\mathrm{T}}=\boldsymbol{x}_{\varepsilon \mathrm{Q}}^{j}{ }^{\mathrm{T}}(\varepsilon=j, k, m, u), \boldsymbol{q}_{\varepsilon}{ }^{\mathrm{T}}=\boldsymbol{x}_{\varepsilon}{ }^{\mathrm{T}} \quad(\varepsilon=1,2, \cdots, n$, and $\varepsilon \neq j, k, m, u)$.

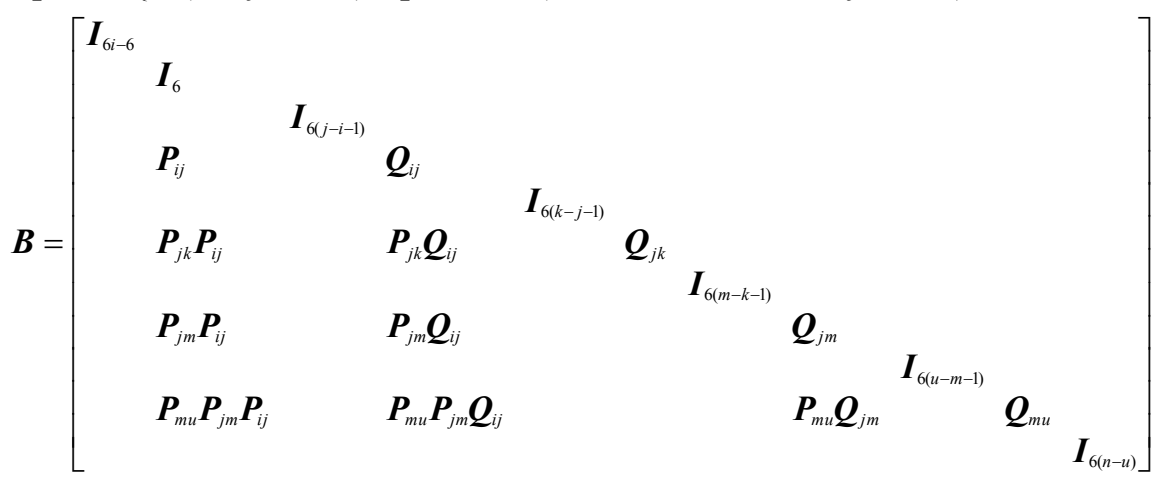

The blank blocks in $\boldsymbol{B}$ are all zero matrices with corresponding dimensions, and the subscript of each identity matrix $\boldsymbol{I}$ indicates its dimension. It can be seen that the overall transform matrix $\boldsymbol{B}$ contains information about all joints and the intrinsic constraint topology in the multibody system.

The independent absolute joint coordinates for the system, i.e., $\hat{\boldsymbol{x}}$, can be written as

$$
\hat{\boldsymbol{x}}=\boldsymbol{W}^{\mathrm{T}} \overline{\boldsymbol{x}}
$$

The dimension-reduction matrix for the system, i.e., $\boldsymbol{W}$, follows

$$
\boldsymbol{W}=\operatorname{diag}\left(\boldsymbol{W}_{1} \boldsymbol{W}_{2} \cdots \boldsymbol{W}_{n}\right)
$$

where $\boldsymbol{W}_{j}=\boldsymbol{D}_{i j}, \boldsymbol{W}_{k}=\boldsymbol{D}_{j k}, \boldsymbol{W}_{m}=\boldsymbol{D}_{k m}, \boldsymbol{W}_{u}=\boldsymbol{D}_{m u}, \boldsymbol{W}_{\varepsilon}=\boldsymbol{I}_{6} \quad(\varepsilon=1,2, \cdots, n$, and $\varepsilon \neq j, k, m, u)$.

Replace $\overline{\boldsymbol{x}}$ by $\boldsymbol{W} \hat{\boldsymbol{x}}$ in Eq.(12), yield

$$
\boldsymbol{x}=\boldsymbol{B} \boldsymbol{W} \hat{\boldsymbol{x}}
$$

Substitute Eq.(13) into Eq.(10), a minimal set of linearized ODEs for the open-loop multibody system can be obtained in terms of absolute joint coordinates

$$
\begin{gathered}
\hat{\boldsymbol{M}} \ddot{\hat{\boldsymbol{x}}}+\hat{\boldsymbol{C}} \dot{\hat{\boldsymbol{x}}}+\hat{\boldsymbol{K}} \hat{\boldsymbol{x}}=\boldsymbol{B} \boldsymbol{W} \boldsymbol{f}_{e} \\
\hat{\boldsymbol{E}}=\boldsymbol{W}^{\mathrm{T}} \boldsymbol{B}^{\mathrm{T}} \boldsymbol{E} \boldsymbol{B} \boldsymbol{W} \quad(\boldsymbol{E}=\boldsymbol{M}, \boldsymbol{K}, \boldsymbol{C})
\end{gathered}
$$

The eigenvalues and eigenvectors of the system can thus be calculated using standard numerical method easily, and consequently, the natural frequencies, the corresponding damping ratio and mode shapes of the system can be obtained.

\section{Numerical experiments}

The proposed algorithm, named as AMVA (Automatic Modeling for Vibration Analysis), 
is implemented in MATLAB. The eigenvalues and eigenvectors are computed using standard LAPACK routines. Open-loop holonomic multibody systems with chain and tree topology are taken as case studies to test the correctness and efficiency of the proposed method.



(a) Chain topology

Body

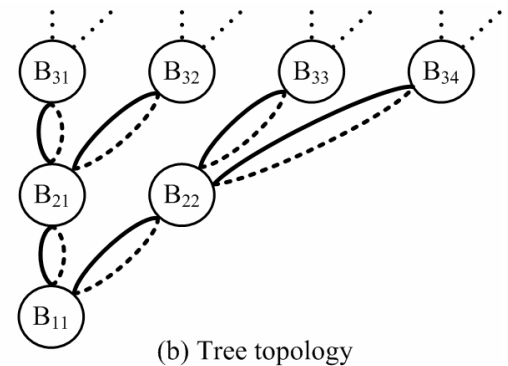

(b) Tree topology

Fig. 4 Sketch map of models used for numerical test

A) Multibody systems with chain topology. As shown in Fig. 4(a), $n$ bodies are connected by joints and spatial spring-dampers in a chain. Parameters for each rigid body $\mathrm{B}_{i}$ and each spring-damper $\mathrm{K}_{i-1, i, 1}$ between $\mathrm{B}_{i}$ and $\mathrm{B}_{i-1}\left(\mathrm{~B}_{0}\right.$ is the ground) are shown in Table $\mathrm{A} 1$ and Table $A 2$ in Appendix, respectively. The position and orientation of joint $J_{i-1, i}$ between $\mathrm{B}_{i}$ and $\mathrm{B}_{i-1}$ are identical to that of spring-damper $\mathrm{K}_{i-1, i, 1}$. Information about each chain topology models, i.e., C100CF1, C200P F1, C100SF1, are listed in Table A3.

B) Multibody systems with tree topology. As shown in Fig. 4(b), the bodies are connected by joints and spatial spring-dampers in the form of binary tree with $N$ layers. The number of bodies and spring-dampers, as well as the number and type of joints for each model, are list in Table A3 in Appendix. There are $n_{j}=2^{j-1}$ bodies in the $j^{\text {th }}$ layer. The parameters for the $i^{\text {th }}$ rigid body in the $j^{\text {th }}$ layer, i.e., $\mathrm{B}_{j i}$, are listed in Table $\mathrm{A} 4$ in Appendix. The parameters for spring-dampers $\mathrm{K}_{p, q}$ between $\mathrm{B}_{j i}$ and $\mathrm{B}_{j+1, m}(m=2 i-1,2 i)$ are listed in Table A5 in Appendix. The position and orientation of joint $\mathrm{J}_{p, q}$ are identical to that of spring-damper $\mathrm{K}_{p, q}$. The rest information about each tree topology models (model name starting with letter ' $\mathrm{L}$ ') are also listed in Table A3.

Normal mode analysis (NMA) and transfer function analysis (TFA) were performed in ADAMS and AMVA for each model. The simulations and computations are performed on a PC-compatible computer with CPU Pentium 4 of $2.0 \mathrm{GHz}$ and memory of 2.0 GB.

For TFA of model L7SF1, a swept sine signal with unit amplitude is used as input force acting on body $\mathrm{B}_{6,32}$ in $Y$-direction, the output is the displacement response in $Y$-direction for the same body. The results for model L7SF1 (as an example) are shown in Fig. 5 and Fig. 6, respectively. Solutions in Fig. 5 show that the results of eigenvalue calculated using AMVA are identical to those of ADAMS. The mean and maximal errors of natural frequencies between the two groups of results are $1.016 \times 10^{-6} \mathrm{~Hz}$ and $5.000 \times 10^{-5} \mathrm{~Hz}$, respectively. The mean and maximal errors of damping ratios of the two groups of results are $1.730 \times 10^{-10}$ and $5.000 \times 10^{-8}$, respectively. Comparisons shown in Fig. 6 indicate that solutions of transfer function computed using AMVA coincide well with those of ADAMS.
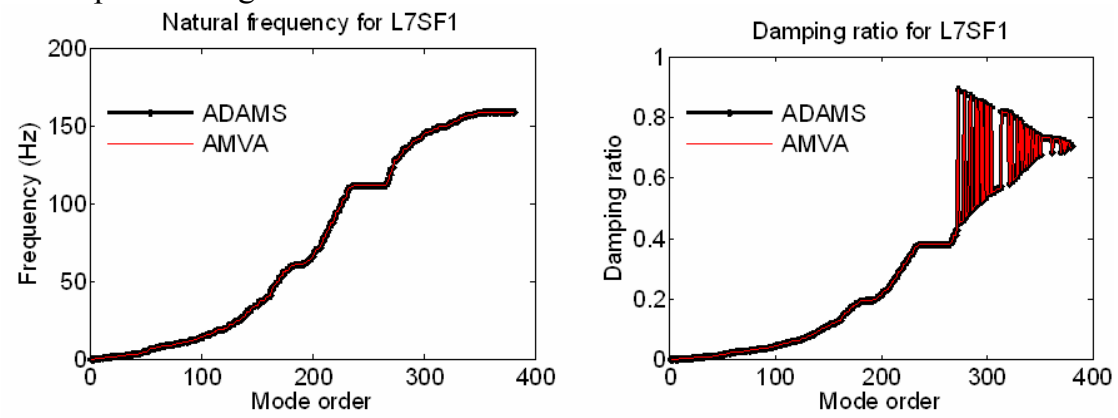

Fig. 5 Comparison of NMA results for model L7SF1 




Fig. 6 Comparison of TFA solutions for model L7SF1

Table 2 Comparison of solution time for different models

\begin{tabular}{ccccc}
\hline \multirow{2}{*}{ Model name } & $\begin{array}{c}\text { Solution time in ADAMS (s) } \\
\text { NMA }\end{array}$ & $\begin{array}{c}\text { Solution time using AMVA (s) } \\
\text { NMA }\end{array}$ & TFA \\
\hline C100CF1 & 17.2 & 17.8 & 3.92 & 4.01 \\
C200PF1 & 52.7 & 54.4 & 7.6 & 7.8 \\
C100SF1 & 55.2 & 59.8 & 14.9 & 15.6 \\
L6SF1 & 15.0 & 17.8 & 3.8 & 4.1 \\
L7SF1 & 109.0 & 115.3 & 20.1 & 25.6 \\
L7CF1 & 33.6 & 33.9 & 5.9 & 6.5 \\
L7RF1 & 16.3 & 17.4 & 2.0 & 2.8 \\
L7PF1 & 17.9 & 18.9 & 1.7 & 2.5 \\
L7CF5 & 273.0 & 275.0 & 6.6 & 7.2 \\
L7RF5 & 132.0 & 134.0 & 2.9 & 3.6 \\
L7PF5 & 137.0 & 139.0 & 2.4 & 3.3 \\
L8CF1 & 333.0 & 354.0 & 39.3 & 38.2 \\
L8RF1 & 119.0 & 111.0 & 11.6 & 11.9 \\
L8PF1 & 120.0 & 127.0 & 9.5 & 10.2 \\
\hline
\end{tabular}

Time consumption for solution both in ADAMS and using AMVA for the aforementioned models are shown in Table 2, respectively. It indicates that the solution time is affected by the number of bodies, the number and type of joints, as well as the number of spring-dampers. Generally, the solution time for NMA and TFA in either tool will increase under any of the following conditions:

a) the number of DOFs increases due to change in type of joints while the number of bodies, joints and spring-dampers remain unchanged, e.g., L7SF1, L7CF1, L7RF1;

b) the number of bodies increases while the number of DOFs and spring-dampers remain unchanged, which means that the number and/or type of joints will change, e.g., C100CF1, C200PF1;

c) the number of spring-dampers increases while the number of bodies, joints and DOFs remain unchanged, e.g., L7CF1 and L7CF5, L7RF1 and L7RF5, L7PF1 and L7PF5.

It can also be concluded that while performing NMA and TFA for the same model, the solution time using AMVA is much less than that in ADAMS. Furthermore, the greater the number of joints, bodies, or spring-dampers is, the more the improvement in computational efficiency is obtained. Especially, although the final equations are identical for two models with the same topology of constraints, e.g., L7CF1 and L7CF5, L7RF1 and L7RF5, L7PF1 and L7PF5, the number of spring-dampers has great influence on the solution time in ADAMS, whereas its influence on the solution time using AMVA is neglectable. 
Comparison indicates that the efficiency of NMA and TFA for large-scale spatial open-loop multibody systems has been considerably improved using the presented algorithm in compare with the commercial package ADAMS. More important, such amelioration will lead to significant improvement of efficiency in the case of optimization of large-scale multibody systems where vibration analysis is required to execute iteratively.

\section{Conclusions}

In this paper, absolute joint coordinates are introduced to efficiently formulate the dynamics of large-scale spatial open-loop holonomic multibody systems with both rotational and translational joints. The relationship between absolute joint coordinates and absolute coordinates is presented as an overall transform matrix, which contains only information about all constraints for an open-loop holonomic multibody system. Equations using absolute joint coordinates are simpler in form than the corresponding equations in absolute coordinates or joint coordinates for open-loop multibody systems, and are easier to be generated and understood than those using indirect coordinates. A minimal set of ODEs, which can be used for linear vibration analysis and control, are formulated via matrix transformation instead of solving constraint equations for open-loop holonomic multibody systems. Extra linearization is unnecessary because the equations are directly linearized in the formulation.

The correctness and efficiency of the proposed method are verified using spatial multibody systems with open-loop topologies, i.e., chain topology and tree topology. The efficiency of linear vibration analysis has been considerablely improved using the presented algorithm in compare with the commercial package ADAMS. In addition, the greater the number of joints, bodies, or spring-dampers is, the more improvement in computational efficiency is achieved. The proposed method shows promise in vibration analysis, control, and optimization, of large-scale spatial open-loop holonomic multibody systems.

\section{Acknowledgments}

The work is partially supported by the National Basic Research Program of China (973 Program, granted No. 2003CB716206), the National Natural Science Foundation of China (NSFC, granted No. 50605025), and the Innovation Fund for Outstanding Scholar of Henan Province (granted No. 0621000300).

Appendix

Table A1 Parameters of body $\mathrm{B}_{i}$ in chain topology models

\begin{tabular}{|c|c|c|c|}
\hline Item & Symbol & Value & Unit \\
\hline Mass & $m$ & 12.25 & $\mathrm{~kg}$ \\
\hline Inertia & {$\left[\begin{array}{llllll}I_{x x} & I_{y y} & I_{z z} & I_{x y} & I_{x z} & I_{y z}\end{array}\right]$} & {$\left[\begin{array}{llllll}1.53 & 4.85 & 4.85 & 0 & 0 & 0\end{array}\right] \times 10^{-2}$} & $\mathrm{~kg} \cdot \mathrm{m}^{2}$ \\
\hline CM position & {$\left[\begin{array}{lll}\underline{x}_{c i} & \underline{y}_{c i} & \underline{Z}_{c i}\end{array}\right]$} & {$[0.2 i-0.100]$} & $\mathrm{m}$ \\
\hline $\mathrm{CM}$ orientation & {$\left[\begin{array}{lll}\underline{\alpha}_{c i} & \underline{\beta}_{c i} & \underline{\gamma}_{c i}\end{array}\right]$} & {$\left[\begin{array}{lll}0 & 0 & 0\end{array}\right]$} & $\mathrm{rad}$ \\
\hline
\end{tabular}

Table A2 Parameters of spring-damper $\mathrm{K}_{i-1, i, 1}$ in chain topology models

\begin{tabular}{|c|c|c|c|}
\hline Item & Symbol & Value & Unit \\
\hline Stiffness & {$\left[\begin{array}{lll}k_{x}^{k} & k_{y}^{k} & k_{z}^{k}\end{array}\right]$} & {$\left[\begin{array}{lll}1.00 & 1.00 & 3.98\end{array}\right] \times 10^{9}$} & $\mathrm{~N} \cdot \mathrm{m}^{-1}$ \\
\hline Torsion stiffness & {$\left[\begin{array}{lll}k_{\alpha}^{k} & k_{\beta}^{k} & k_{\gamma}^{k}\end{array}\right]$} & {$\left[\begin{array}{lll}2.49 \times 10^{8} & 2.49 \times 10^{8} & 1.73 \times 10^{6}\end{array}\right]$} & $\mathrm{N} \cdot \mathrm{m} \cdot \mathrm{rad}^{-1}$ \\
\hline Damping & {$\left[\begin{array}{lll}c_{x}^{k} & c_{y}^{k} & c_{z}^{k}\end{array}\right]$} & {$\left[\begin{array}{lll}10 & 10 & 10\end{array}\right]$} & $\mathrm{N} \cdot \mathrm{s} \cdot \mathrm{m}^{-1}$ \\
\hline Torsion damping & {$\left[\begin{array}{ccc}c_{\alpha}^{k} & c_{\beta}^{k} & c_{\gamma}^{k}\end{array}\right]$} & {$\left[\begin{array}{lll}2.86 \times 10^{2} & 2.86 \times 10^{2} & 5.73 \times 10^{1}\end{array}\right]$} & $\mathrm{N} \cdot \mathrm{m} \cdot \mathrm{s} \cdot \mathrm{rad}^{-1}$ \\
\hline Position & {$\left[\underline{x}_{i-1, i} \quad \underline{y}_{i-1, i} \underline{z}_{i-1, i}\right]$} & {$[0.2 i-0.200]$} & $\mathrm{m}$ \\
\hline Orientation & {$\left[\underline{\alpha}_{i-1, i} \underline{\beta}_{i-1, i} \underline{\gamma}_{i-1, i}\right]$} & {$\left[\begin{array}{lll}\pi / 2 & \pi / 2 & 0\end{array}\right]$} & $\mathrm{rad}$ \\
\hline
\end{tabular}


Table A3 Details for different models

\begin{tabular}{ccccc}
\hline Model name & No. bodies & Joint type & DOFs & No. spring-dampers \\
\hline C100CF1 & 100 & cylindrical & 200 & 1 \\
C200PF1 & 200 & prismatic & 200 & 1 \\
C100SF1 & 100 & spherical & 300 & 1 \\
L6SF1 & 63 & spherical & 189 & 1 \\
L7SF1 & 127 & spherical & 381 & 1 \\
L7CF1 & 127 & cylindrical & 254 & 1 \\
L7RF1 & 127 & revolute & 127 & 1 \\
L7PF1 & 127 & prismatic & 127 & 1 \\
L7CF5 & 127 & cylindrical & 254 & 5 \\
L7RF5 & 127 & revolute & 127 & 5 \\
L7PF5 & 127 & prismatic & 127 & 5 \\
L8CF1 & 255 & cylindrical & 510 & 1 \\
L8RF1 & 255 & revolute & 255 & 1 \\
L8PF1 & 255 & prismatic & 255 & 1 \\
\hline
\end{tabular}

Table A4 Parameters of body $\mathrm{B}_{i i}$ in tree topology models

\begin{tabular}{|c|c|c|c|}
\hline Item & Symbol & Value & Unit \\
\hline Mass & $m$ & 1.00 & $\mathrm{~kg}$ \\
\hline Inertia & {$\left[\begin{array}{llllll}I_{x x} & I_{y y} & I_{z z} & I_{x y} & I_{x z} & I_{y z}\end{array}\right]$} & {$\left[\begin{array}{llllll}1.00 & 1.00 & 1.00 & 0 & 0 & 0\end{array}\right]$} & $\mathrm{kg} \cdot \mathrm{m}^{2}$ \\
\hline CM position & {$\left[\begin{array}{lll}\underline{x}_{c j i} & \underline{y}_{c j i} & \underline{z}_{c j i}\end{array}\right]$} & {$\left[\begin{array}{lll}i & j & 0\end{array}\right]$} & $\mathrm{m}$ \\
\hline $\mathrm{CM}$ orientation & {$\left[\begin{array}{lll}\underline{\alpha}_{c j i} & \underline{\beta}_{c j i} & \underline{\gamma}_{c j i}\end{array}\right]$} & {$\left[\begin{array}{lll}0 & 0 & 0\end{array}\right]$} & $\mathrm{rad}$ \\
\hline \multicolumn{4}{|c|}{ Table A5 Parameters of spring-damper $\mathrm{K}_{p, q}$ in tree topology models } \\
\hline Item & Symbol & Value & Unit \\
\hline Stiffness & {$\left[\begin{array}{lll}k_{x}^{k} & k_{y}^{k} & k_{z}^{k}\end{array}\right]$} & {$\left[\begin{array}{lll}1.00 & 1.00 & 1.00\end{array}\right] \times 10^{4}$} & $\mathrm{~N} \cdot \mathrm{m}^{-1}$ \\
\hline Torsion stiffness & {$\left[\begin{array}{lll}k_{\alpha}^{k} & k_{\beta}^{k} & k_{\gamma}^{k}\end{array}\right]$} & {$\left[\begin{array}{lll}5.73 & 5.73 & 5.73\end{array}\right] \times 10^{5}$} & $\mathrm{~N} \cdot \mathrm{m} \cdot \mathrm{rad}^{-1}$ \\
\hline Damping & {$\left[\begin{array}{lll}c_{x}^{k} & c_{y}^{k} & c_{z}^{k}\end{array}\right]$} & {$\left[\begin{array}{lll}10 & 10 & 10\end{array}\right]$} & $\mathrm{N} \cdot \mathrm{s} \cdot \mathrm{m}^{-1}$ \\
\hline Torsion damping & {$\left[\begin{array}{lll}c_{\alpha}^{k} & c_{\beta}^{k} & c_{\gamma}^{k}\end{array}\right]$} & {$\left[\begin{array}{lll}5.73 & 5.73 & 5.73\end{array}\right] \times 10^{2}$} & $\mathrm{~N} \cdot \mathrm{m} \cdot \mathrm{s} \cdot \mathrm{rad}^{-1}$ \\
\hline Position & {$\left[\begin{array}{lll}\underline{x}_{p, q} & \underline{y}_{p, q} & \underline{z}_{p, q}\end{array}\right]$} & {$[(i+n) / 2 \quad j+0.50]$} & $\mathrm{m}$ \\
\hline Orientation & {$\left[\begin{array}{lll}\underline{\alpha}_{p, q} & \underline{\beta}_{p, q} & \underline{\gamma}_{p, q}\end{array}\right]$} & {$\left[\begin{array}{lll}0 & 0 & \arctan (2 m-2 i)\end{array}\right]$} & $\mathrm{rad}$ \\
\hline
\end{tabular}

\section{References}

1. Schiehlen, W., Guse, N., Seifried, R., Multibody dynamics in computational mechanics and engineering applications, Comput. Methods Appl. Mech. Engrg., Vol.195, pp.5509-5522, 2006.

2. Laulusa, A., Bauchau, O.A., Review of classical approaches for constraint enforcement in multibody systems, ASME J. Comput. Nonlin. Dyn., Vol.3, p.011004, 2008.

3. Bauchau, O.A., Laulusa, A., Review of contemporary approaches for constraint enforcement in multibody systems, ASME J. Comput. Nonlin. Dyn., Vol.3, p.011005, 2008.

4. Samin, J.C., Brüls, O., Collard, J.F., Sass, L., Fisette, P., Multiphysics modeling and optimization of mechatronic multibody systems, Multibody Syst. Dyn., Vol.18, pp.345-373, 2007.

5. Kurdila, A., Papastavridis, J.G., and Kamat, M.P., Role of Maggi's equations in computational methods for constrained multibody systems, J. Guid., Control, Dyn., Vol.13, No.1, pp.113-120, 1990.

6. Gear, C.W., Leimkuhler, B., and Gupta, G.K., Automatic integration of Euler-Lagrange equations with constraints, J. Comput. Appl. Math., Vol.12\&13, pp.77-90, 1985.

7. Borri, M., Bottasso, C.L., and Mantegazza, P., Acceleration projection method in multibody dynamics, Eur. J. Mech., A/Solids, Vol.11, No.3, pp.403-418, 1992.

8. Udwadia, F.E., and Kalaba, R.E., What is the general form of the explicit equations of motion for constrained mechanical system, ASME J. Appl. Mech., Vol.69, pp.335-339, 2002. 
9. Kang, J.S., Bae, S., Lee, J.M., Tak, T.O., Force equilibrium approach for linearization of constrained mechanical system dynamics, ASME J. Mech. Des., Vol.125, pp.143-149, 2003.

10. Minaker B., Frise, P., Linearizing the equations of motion for multibody systems using an orthogonal complement method, J. Vibr. Control, Vol.11, pp.51-66, 2005.

11. Negrut D., Ortiz, J.L., A practical approach for the linearization of the constrained multibody dynamics equations, ASME J. Comput. Nonlin. Dyn., Vol.1, pp.230-239, 2006.

12. Pott, A., Kecskeméthy, A., Hiller, M., A simplified force-based method for the linearization and sensitivity analysis of complex manipulation systems. Mech. Mach. Theory, Vol.42, pp.1445-1461, 2007.

13. McPhee, J.J., Redmond, S.M, Modelling multibody systems with indirect coordinates, Comput. Methods Appl. Mech. Engrg., Vol.195, pp.6942-6957, 2006.

14. Haug, E.J., Computer-aided kinematics and dynamics of mechanical systems, Allyn and Bacon, Boston, MA, 1989

15. Wittenburg, J., Dynamics of multibody systems, $2^{\text {nd }}$ ed., Springer, Berlin, 2008.

16. Huston, R.L. et al, Use of absolute coordinates in computational multibody dynamics, Comput. Struct., Vol.52, pp.17-25, 1994.

17. Fayet, M., Pfister, F., Analysis of multibody systems with indirect coordinates and global inertia tensors, Eur. J. Mech., A/Solids, Vol.13, No. 3, pp.431-457, 1994.

18. McPhee, J.J., Automatic generation of motion equations for planar mechanical systems using the new set of "branch coordinates", Mech. Mach. Theory, Vol.33, No. 6, pp.805-823, 1998.

19. McPhee, J.J., Schmitke, C., Redmond, S.M, Dynamic modelling of mechatronic multibody systems with symbolic computing and linear graph theory, Math. Comput. Modell. Dyn. Syst., Vol.10, No. 1, pp.1-23, 2004.

20. Richard, M.J., McPhee, J.J., Anderson, R.J., Computerized generation of motion equations using variational graph-theoretic methods, Appl. Math. Comput. Vol.192, pp.135-156, 2007.

21. Fisette, P., Johnson, D.A., Samin, J.C., A fully symbolic generation of the equations of motion of multibody systems containing flexible beams, Comput. Methods Appl. Mech. Engrg., Vol.142, pp.123-152, 1997.

22. Forehand, D.I.M., Khanin, R., Cartmell, M.P., A Lagrangian multibody code for deriving the symbolic state-space equations of motion for open-loop systems containing flexible beams, Math. Comput. Simulat., Vol.67, pp.85-98, 2004.

23. Park, F.C., Choi, J., Ploen, S.R., Symbolic formulation of closed chain dynamics in independent coordinates, Mech. Mach. Theory, Vol.34, pp.731-751, 1999.

24. Samin J.C., Fisette, P., Symbolical modelling of multibody systems, Kluwer, Dordrecht, 2003.

25. Cui K., Haque, I., Symbolic equations of motion for hybrid multibody systems using a matrix-vector formulation, Mech. Mach. Theory, Vol.32, No.6, pp.743-763, 1997. 